28 May 2003

physics/0305122

\title{
Lorentz Invariance of Neutrino Oscillations
}

\author{
C. Giunti \\ INFN, Sezione di Torino, and Dipartimento di Fisica Teorica, \\ Università di Torino, Via P. Giuria 1, I-10125 Torino, Italy
}

\begin{abstract}
It is shown that, in spite of the appearances, the standard expression for the oscillation probability of ultrarelativistic neutrinos is Lorentz invariant.
\end{abstract}

Flavor is the quantum number that distinguishes the different types of quarks and leptons. It is a Lorentz-invariant quantity. For example, an electron is seen as an electron by any observer, never as a muon. Therefore, the probability of flavor neutrino oscillations must be Lorentz invariant (as already remarked in Ref. 1]). However, it may appear that the standard expression for the probability of $\nu_{\alpha} \rightarrow \nu_{\beta}$ transitions in vacuum ${ }^{1}$,

$$
P_{\nu_{\alpha} \rightarrow \nu_{\beta}}(E, L)=\sum_{k}\left|U_{\alpha k}\right|^{2}\left|U_{\beta k}\right|^{2}+2 \operatorname{Re} \sum_{k>j} U_{\alpha k}^{*} U_{\beta k} U_{\alpha j} U_{\beta j}^{*} e^{-i \Delta \phi_{k j}(E, L)},
$$

with the phase differences

$$
\Delta \phi_{k j}(E, L)=\frac{\Delta m_{k j}^{2} L}{2 E}
$$

is not Lorentz invariant. In Eq. (11) $U$ is the mixing matrix that connects the flavor neutrino fields $\nu_{\alpha}(\alpha=e, \mu, \tau)$ with the massive neutrino fields $\nu_{k}(k=1,2,3)^{2}, L$ is the distance between the neutrino source and the neutrino detector, and $E$ is the neutrino energy (see Refs. [2, 3] ). In Eq. (2) $\Delta m_{k j}^{2} \equiv m_{k}^{2}-m_{j}^{2}$ is the difference between the squared-masses of $\nu_{k}$ and $\nu_{j}$.

Let us assume that Eq. (11) is valid in the inertial system $\mathcal{O}$ with time axis $t$ and the $x$ axis in the direction of neutrino propagation. Consider another inertial system $\mathcal{O}^{\prime}$ with axes $x^{\prime}, t^{\prime}$ moving with respect to $\mathcal{O}$ with velocity $v$ in the $x$ direction. The Lorentz transformations

\footnotetext{
${ }^{1}$ In the simplest case of two-neutrino mixing with $\nu_{\alpha}=\cos \vartheta \nu_{1}+\sin \vartheta \nu_{2}$ and $\nu_{\beta}=-\sin \vartheta \nu_{1}+\cos \vartheta \nu_{2}$, the transition probability in Eq. (11) reduces to the well known expression $P_{\nu_{\alpha} \rightarrow \nu_{\beta}}(E, L)=\sin ^{2} 2 \vartheta \sin ^{2}\left(\frac{\Delta m^{2} L}{4 E}\right)$.

${ }^{2}$ If the number of massive neutrinos is $N>3$, the indices $k, j$ run from 1 to $N$ and $\alpha, \beta=$ $e, \mu, \tau, s_{1}, \ldots, s_{N-3}$, with the indices $s_{1}, \ldots, s_{N-3}$ denoting $N-3$ sterile neutrino fields (see Refs. [2, 3]).
} 
of space and time intervals are ${ }^{3}$

$$
\begin{aligned}
& \Delta x^{\prime}=\gamma(\Delta x-v \Delta t), \\
& \Delta t^{\prime}=\gamma(-v \Delta x+\Delta t),
\end{aligned}
$$

with $\gamma \equiv\left(1-v^{2}\right)^{-1 / 2}$. The length $\Delta x^{\prime}$ in the system $\mathcal{O}^{\prime}$ of an object with length $\Delta x$ in the system $\mathcal{O}$ is calculated at equal times in the system $\mathcal{O}^{\prime}$ :

$$
\Delta t^{\prime}=0 \quad \Longrightarrow \quad \Delta t=v \Delta x \quad \Longrightarrow \quad \Delta x^{\prime}=\gamma\left(1-v^{2}\right) \Delta x=\frac{\Delta x}{\gamma} .
$$

This is the well-known Lorentz contraction of distances. The Lorentz-contracted distance between neutrino source and detector measured in the system $\mathcal{O}^{\prime}$ is given by

$$
L^{\prime}=\frac{L}{\gamma}
$$

On the other hand, the Lorentz transformations of momentum and energy are

$$
\begin{aligned}
& p^{\prime}=\gamma(p-v E) \\
& E^{\prime}=\gamma(-v p+E) .
\end{aligned}
$$

In the massless limit

$$
E=p \quad \Longrightarrow \quad E^{\prime}=p^{\prime}=\gamma(1-v) E
$$

It is clear that if the values of $L^{\prime}$ and $E^{\prime}$ in Eqs. (15) and (7) were correct for the calculation of the oscillation probability in the system $\mathcal{O}^{\prime}$, the resulting oscillation probability $P_{\nu_{\alpha} \rightarrow \nu_{\beta}}\left(E^{\prime}, L^{\prime}\right)$ would be different from $P_{\nu_{\alpha} \rightarrow \nu_{\beta}}(E, L)$, leading to an absurd violation of the Lorentz invariance of flavor.

A possible objection to this reasoning is that the phase differences in Eq. (21) are valid only in the reference frame in which the source and detector are at rest. The answer to this objection is that the expression in Eq. (22) is derived without any assumption of a special reference frame, and hence it must be valid in any frame. Indeed, the phase differences in Eq. (2) follow from a simple relativistic approximation of the explicitly Lorentz invariant phases

$$
\phi_{k}(T, L)=E_{k} T-p_{k} L,
$$

where $T$ is the time of propagation of the neutrino from the source to the detector, $E_{k}$ and $p_{k}$ are the energy and momentum of $\nu_{k}$. For $T=L$, corresponding to the leading order contribution to the phase of ultrarelativistic neutrinos (see Ref. [1]), we have

$$
\phi_{k}(T=L)=\left(E_{k}-p_{k}\right) L=\frac{E_{k}^{2}-p_{k}^{2}}{E_{k}+p_{k}} L=\frac{m_{k}^{2}}{E_{k}+p_{k}} L \simeq \frac{m_{k}^{2}}{2 E} L,
$$

where we have used the relativistic dispersion relation $E_{k}^{2}=p_{k}^{2}+m_{k}^{2}$, and $E$ is the neutrino energy in the limit $m_{k} \rightarrow 0$. The difference of the phases in Eq. (91) is the phase difference

\footnotetext{
${ }^{3}$ We use natural units, with $c=1$.
} 
in Eq. (2). From this simple derivation in which we did not make any assumption on the reference frame, it is clear that the phases (9) and the phase differences (2) are valid in any frame and should be Lorentz invariant as the phase (8) from which they are derived.

The above derivation helps in understanding the solution of the problem: the crucial point is the approximation $T=L$, which means that $L$ is not the equal-time distance between source and detector, but the distance traveled by the neutrino in the time $T$. In other words, in any system $L$ is the spatial distance between the space-time events of neutrino production and detection.

If in the system $\mathcal{O}$ we have $\Delta x=\Delta t$, from Eqs.(3) in the system $\mathcal{O}^{\prime}$ we have

$$
\Delta x^{\prime}=\Delta t^{\prime}=\gamma(1-v) \Delta x
$$

Therefore, the correct transformation for the distance in the phases is not given by the Lorentz contraction formula in Eq. (15), but by

$$
L^{\prime}=\gamma(1-v) L
$$

Confronting this expression with the transformation of energy in Eq. (17), it is clear that the ratio $L / E$ is Lorentz invariant, as well as the phases in Eqs. (9) and (2), and the flavor transition probability in Eq. (11).

A simple example can show clearly that the Lorentz-contracted source-detector distance (5) calculated at equal times in the system $\mathcal{O}^{\prime}$ does not correspond to the distance traveled by the neutrino. Consider the source and detector at rest in the system $\mathcal{O}$. Then in the system $\mathcal{O}^{\prime}$ the detector is moving with velocity $-v$ along the $x$ axis. Since the detector moves after the propagating neutrino has left the source, the spatial distance traveled by the neutrino is shorter than the instantaneous source-detector distance.

The correct transformation law of the propagation distance in Eq. (11) is illustrated by the Minkowski diagram in Fig. 1, in which one can clearly see that in a system in which source and detector are in motion the spatial distance between the two space-time events of neutrino production and detection is different from the instantaneous source-detector distance. The two distances coincide only in the system $\mathcal{O}$, in which source and detector are at rest.

Finally, one can ask what happens if instead of considering the source and detector at rest in the system $\mathcal{O}$, we consider a case in which source and detector are in relative motion. Since the oscillation probability is measured by the detector, the velocity of the source with respect to the detector does not matter ${ }^{4}$. The system $\mathcal{O}$ in which the distance $L$ covered by the neutrino coincides with the instantaneous distance between source and detector at the time of neutrino emission is always the rest system of the detector. In Fig. 1 the world-line of the detector is constrained to be the vertical line passing through the space-time event $D$ of neutrino detection, but the world-line of the source can be any time-like line passing through the space-time event $P$ of neutrino production.

\footnotetext{
${ }^{4}$ Of course, the velocity of the source must be taken into account in the calculation of the neutrino energy. For example, if the source is a decaying pion, the neutrino energy depends on the velocity of the pion.
} 


\section{Acknowledgments}

I would like to thank the School of Natural Sciences, Institute for Advanced Study, Princeton for kind hospitality during the completion of this work.

\section{References}

[1] C. Giunti and C. W. Kim, Found. Phys. Lett. 14, 213 (2001), hep-ph/0011074.

[2] S. M. Bilenky, C. Giunti, and W. Grimus, Prog. Part. Nucl. Phys. 43, 1 (1999), hep-ph/9812360.

[3] M. Gonzalez-Garcia and Y. Nir, Rev. Mod. Phys. 75, 345 (2003), hep-ph/0202058. 


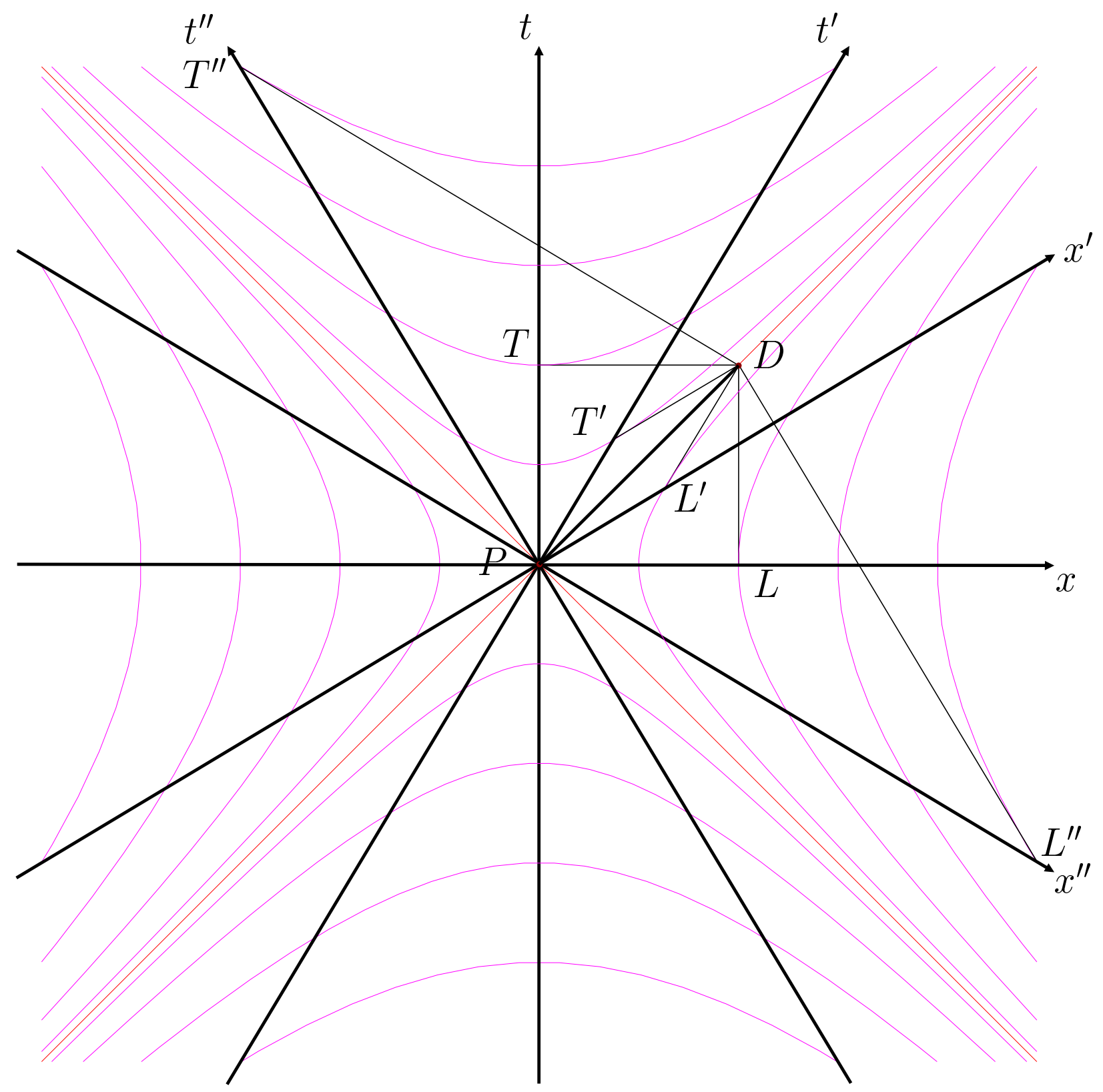

Figure 1: Minkowski diagram showing the distance and time between the space-time events of neutrino production $P$ and detection $D$ measured in three coordinate systems: $\mathcal{O}$ with axes $x, t ; \mathcal{O}^{\prime}$ with axes $x^{\prime}, t^{\prime}$ moving with respect to $\mathcal{O}$ with velocity $v=3 / 5$ in the $x$ direction along neutrino propagation; $\mathcal{O}^{\prime \prime}$ with axes $x^{\prime \prime}, t^{\prime \prime}$ moving with respect to $\mathcal{O}$ with velocity $v=-3 / 5$ in the $x$ direction. The $x^{\prime}\left(x^{\prime \prime}\right)$ axis is inclined with respect to the $x$ axis by an angle $\arctan 3 / 5(-\arctan 3 / 5)$; the $t^{\prime}\left(t^{\prime \prime}\right)$ axis is inclined with respect to the $t$ axis by an angle $-\arctan 3 / 5(\arctan 3 / 5)$. The hyperbolas $t^{2}-x^{2}=t^{\prime 2}-x^{\prime 2}=t^{\prime \prime 2}-x^{\prime \prime 2}=$ constant fix the scale on the axes. One can see that $L^{\prime}=L / 2$ and $L^{\prime \prime}=2 L$, in perfect agreement with Eq. (11). 